\title{
Comparison of phosphorescent agents for noninvasive sensing of tumor oxygenation via Cherenkov-excited luminescence imaging
}

Jennifer R. Shell

Ethan P. LaRochelle

Petr Bruza

Jason R. Gunn

Lesley A. Jarvis

David J. Gladstone

Brian W. Pogue 


\title{
Comparison of phosphorescent agents for noninvasive sensing of tumor oxygenation via Cherenkov-excited luminescence imaging
}

\author{
Jennifer R. Shell, ${ }^{a, *, \dagger}$ Ethan P. LaRochelle, ${ }^{a, \dagger}$ Petr Bruza, ${ }^{a}$ Jason R. Gunn, ${ }^{a}$ Lesley A. Jarvis, \\ David J. Gladstone, ${ }^{a, b}$ and Brian W. Pogue ${ }^{a}$ \\ ${ }^{a}$ Dartmouth College, Thayer School of Engineering, Hanover, New Hampshire, United States \\ bDartmouth College, Geisel School of Medicine, Hanover, New Hampshire, United States
}

\begin{abstract}
Cherenkov emission generated in tissue during radiotherapy can be harnessed for the imaging biochemistry of tissue microenvironments. Cherenkov-excited luminescence scanned imaging (CELSI) provides a way to optically and noninvasively map oxygen-related signals, which is known to correlate to outcomes in radiotherapy. Four candidate phosphorescent reagents PtG4, MM2, $\operatorname{Ir}(\mathrm{btb})_{2}$ (acac), and MitolD were studied for oxygen sensing, testing in a progressive series of (a) in solution, (b) in vitro, and (c) in subcutaneous tumors. In each test, the signal strength and response to oxygen were assessed by phosphorescence intensity and decay lifetime measurement. MM2 showed the most robust response to oxygen changes in solution, followed by PtG4, $\operatorname{Ir}(\mathrm{btb})_{2}(\mathrm{acac})$, and MitolD. However, in PANC-1 cells, their oxygen responses differed with $\operatorname{Ir}(\mathrm{btb})_{2}(\mathrm{acac})$ exhibiting the largest phosphorescent intensity change in response to changes in oxygenation, followed by PtG4, MM2, and MitolD. In vivo, it was only possible to utilize $\operatorname{Ir}(\mathrm{btb})_{2}$ (acac) and PtG4, with each being used at nanomole levels, to determine signal strength, lifetime, and $\mathrm{pO}_{2}$. Oxygen sensing with CELSI during radiotherapy is feasible and can estimate values from $1 \mathrm{~mm}$ regions of tissue when used in the configuration of this study. PtG4 was the most amenable to in vivo sensing on the timescale of external beam LINAC x-rays. $\odot$ The Authors. Published by SPIE under a Creative Commons Attribution 4.0 Unported License. Distribution or reproduction of this work in whole or in part requires full attribution of the original publication, including its DOI. [DOI: 10.1117/1.JBO.24.3.036001]
\end{abstract}

Keywords: tumor hypoxia; Cherenkov radiation; phosphorescence; optical imaging.

Paper 180604R received Oct. 31, 2018; accepted for publication Feb. 1, 2019; published online Mar. 4, 2019.

\section{Introduction}

Extent of oxygenation in tumors is a known indicator of the success of radiation therapy, partly due to the oxygen enhancement ratio, ${ }^{1}$ as well as due to oxygen being a surrogate marker for other features of tumor aggressiveness. ${ }^{2}$ Therefore, monitoring of tumor oxygenation during fractionated radiation therapy would be advantageous to gauge the likelihood of response or even to estimate if treatment plan alternations such as a boost to hypoxic areas might be beneficial. Oxygen electrodes have been utilized to measure hypoxia. ${ }^{3-5}$ While this method allows direct measurement of oxygen levels in tumors, it is invasive and is limited to tumors that are easily accessible. Tumor biopsies with indirect measurements for hypoxia, including pimonidazole staining and immunostaining for HIF- $1 \alpha$, provide valuable information of individual tumor microenvironments, ${ }^{6-10}$ but these methods are also invasive and do not provide real-time measurement of tumor oxygenation levels. Blood oxygen level-dependent magnetic resonance imaging (BOLDMRI) ${ }^{11,12}$ electron paramagnetic resonance oximetry, ${ }^{13,14}$ and near-infrared spectroscopic tomography ${ }^{15}$ provide real-time information based on hemoglobin saturation. Positron emission tomography also measures real-time levels of hypoxia, utilizing ${ }^{18}$ F-labeled nitroimidazole derivatives, whose luminescence are dependent on oxygen levels. ${ }^{16,17}$ Despite the potential value, there has not been any clinical convergence on a method for

*Address all correspondence to Jennifer R. Shell, E-mail: jennifer.r.shell@ dartmouth.edu

†Jennifer R. Shell and Ethan P. LaRochelle contributed equally to this work. imaging tumor oxygenation that is noninvasive, precise, and quantitative. In this study, oxygen-related mapping in tumors is demonstrated with Cherenkov-excited luminescence scanned imaging (CELSI), which uses the inherent delivery of radiation to get maps of oxygen-dependent luminescence signals from injected chemical sensors. Current reagents utilized for phosphorescent sensing of oxygen that can be utilized for noninvasive determination of oxygen content [PtG4, MM2, $\operatorname{Ir}(\mathrm{btb})_{2}$ (acac), and MitoID] are evaluated.

Radiation treatment with MV photons or $\mathrm{MeV}$ electrons causes the production of Cherenkov light in tissue. This optical emission occurs when charged primary or secondary electrons pass through the dielectric medium, such as tissue, at a velocity greater than the speed of light. This optical signal has been imaged to visualize surface dose in radiation therapy. ${ }^{18-20}$ In addition, early pilot studies in tissue phantoms and individual animals have shown utilization of Cherenkov emission as the excitation source in imaging applications, including the detection of fluorophores and phosphors in conjunction with radiation therapy. ${ }^{21}$ The potential to sense tumor oxygenation through CELSI with the phosphorescent reagent PtG4 has been shown by us, because the PtG4 phosphorescence is quenched in the presence of oxygen, reducing the observed lifetime of emission. In the presence of ambient oxygen pressure $\left(\mathrm{pO}_{2}\right)$, the PtG4 phosphorescence lifetime is $16.9 \mu \mathrm{s}$, and in low $\mathrm{pO}_{2}$ it is $47 \mu \mathrm{s}^{22,23}$

In this study, this agent was compared to other oxygenquenched chemical agents, and each was examined for its potential to sense tumor hypoxia by directly measuring $\mathrm{pO}_{2}$ levels in vivo with mice bearing subcutaneous tumors. ${ }^{24}$ At the end of the 
study, the spatial confidence in mapping $\mathrm{pO}_{2}$ was estimated, using doses typical of fractionated radiotherapy.

\section{Methods and Materials}

\subsection{Chemicals}

PtG4 (platinum II G4) was provided by Sergei Vinogradov and colleagues. ${ }^{23} \mathrm{MM} 2$ was purchased from Luxcel Bioscience, Cork, Ireland, ${ }^{25}$ bis(2-phenylbenzothiazolato)-(acetylacetonate)iridium(III) $\left[\operatorname{Ir}(\mathrm{btb})_{2}(\mathrm{acac})\right]^{26}$ was purchased from Sigma Aldrich, Saint Louis, Missouri, and Mito-ID intracellular oxygen sensor was purchased from Enzo Life Sciences, Farmingdale, New York. MDA-MB-231 luc-D3H2LN cells were obtained from Perkin Elmer (Waltham, Massachusetts) and PANC-1 cells were obtained from ATCC (CRL-1469, Manassas, Virginia). Dulbecco Modified Eagle Medium (DMEM), fetal bovine serum (FBS), phosphate buffered saline (PBS), and $0.05 \%$ trypsin were purchased from Thermo Fisher Scientific (Waltham, Massachusetts). Molecular Devices Gemini XS (Sunnyvale, California) was utilized for fluorescence plate reader measurements, Agilent Cary $50 \mathrm{UV}$-Vis (Santa Clara, California) was utilized for absorbance spectra, and Horiba Fluorolog-3 (Edison, New Jersey) was utilized for emission spectra. Fluorescence microscopy was performed on a Zeiss LSM 800 (Jena, Germany) confocal microscope.

\subsection{Assessment of Absorbance and Fluorescence Emission Spectra}

The absorbance and fluorescence emission spectra of PtG4 $\left(1 \mu \mathrm{M}, \quad \lambda_{\mathrm{ex}}=435 \mathrm{~nm}\right.$, and $\left.\lambda_{\mathrm{em}}=772 \mathrm{~nm}\right), \operatorname{Ir}(\mathrm{btb})_{2}(\mathrm{acac})$ $\left(1 \mu \mathrm{M}, \lambda_{\mathrm{ex}}=337,480 \mathrm{~nm}\right.$, and $\left.\lambda_{\mathrm{em}}=620 \mathrm{~nm}\right), \mathrm{MM} 2(1 \mu \mathrm{M}$, $\lambda_{\mathrm{ex}}=400 \mathrm{~nm}$, and $\left.\lambda_{\mathrm{em}}=650 \mathrm{~nm}\right)$, and MitoID $(1 \mu \mathrm{M}$, $\lambda_{\mathrm{ex}}=380 \mathrm{~nm}$, and $\lambda_{\mathrm{em}}=650 \mathrm{~nm}$ ) were measured as validation prior to employing these reagents in cell and in vivo studies.

\subsection{Cell Culture}

PANC-1 cells and MDA-MB-231 luc-D3H2LN cells were grown in DMEM with $10 \% \mathrm{FBS}$ in an incubator at $37^{\circ} \mathrm{C}$ with $5 \% \quad \mathrm{CO}_{2}$, and $100 \%$ humidity and divided utilizing $0.05 \%$ trypsin when desired confluency was reached.

\subsection{Fluorescence Assay In Vitro}

PANC-1 cells were seeded in black 96-well plates with a clear bottom at 5000 cells/well. MM2, $\operatorname{Ir}(\mathrm{btb})_{2}(\mathrm{acac})$, and MitoID $(10,20,30 \mu \mathrm{g} / \mathrm{mL}$ in PBS $)$ were added to the cells and allowed to incubate for $24 \mathrm{~h}$ at $37^{\circ} \mathrm{C}$. Media containing the reagents were removed and cells were rinsed with PBS. 10\% FBS in PBS with or without glucose oxidase catalase oxygen scavenger $(100 \mathrm{nM}$ glucose oxidase, $1.5 \mu \mathrm{M}$ catalase, and $56 \mathrm{mM}$ glucose) was added after rinsing and the fluorescence intensity was analyzed with an excitation of $380 \mathrm{~nm}$ and emission of $660 \mathrm{~nm}$. For limits of detection studies, 20, 2, 0.2, and $0.02 \mu \mathrm{g} / \mathrm{mL}$ of MM2 and $\operatorname{Ir}(\mathrm{btb})_{2}(\mathrm{acac})$ were utilized in the same fashion as described above. A student's t-test was utilized to assess differences in fluorescence between oxygenated versus oxygen scavenged conditions.

\subsection{Cherenkov Imaging}

Cherenkov emission was induced by a linear accelerator (Varian LINAC 2100CD, Palo Alto, California). The imaging system consisted of a time-gated intensified CCD camera (ICCD, PIMAX4 1024i, Princeton Instruments) and a commercial lens (Canon EF $135 \mathrm{~mm} \mathrm{f/2L} \mathrm{USM).} \mathrm{The} \mathrm{camera} \mathrm{was} \mathrm{focused} \mathrm{on}$ a mirror that reflected the imaging field $\sim 1 \mathrm{~m}$ away. The time-gated ICCD camera was synchronized to the radiation pulses ( $\sim 3.25 \mu \mathrm{s}$ duration and $360 \mathrm{~Hz}$ repetition rate) with the intensifier set as $\times 100$ and turned on at a 4.26- or $1000-\mu$ s gate delay following each radiation pulse for phosphorescence or background measurement, and luminescence generated during 85.60- $\mu$ s gate width (PtG4 and MM2), or $7.77 \mu \mathrm{s}$ $\left[\operatorname{Ir}(\mathrm{btb})_{2}(\mathrm{acac})\right]$ was integrated via this ICCD. Images of the luminescence at different delay times between LINAC pulse and phosphorescence emission were acquired to construct emission lifetime. To maximize signal and minimize background interference, the room lights were shut off throughout these studies, and all lights in the room were masked off with black cloth and black tape.

\subsection{CEL Studies In Vitro}

Concentrations of 20, 2, 0.2 , and $0.02 \mu \mathrm{g} / \mathrm{mL}$ of $\mathrm{MM} 2$, $\operatorname{Ir}(\mathrm{btb})_{2}$ (acac), MitoID, and PtG4 were utilized 96-well plate as above. For solution studies, $300 \mu \mathrm{L}$ of each solution with and without oxygen scavenger were utilized. For cellular studies, PANC-1 cells were seeded in black 96 -well plates with a clear bottom at 5000 cells/well. The reagents were added to the cells and allowed to incubate for $24 \mathrm{~h}$ at $37^{\circ} \mathrm{C}$. Media containing the reagents were removed and cells were rinsed with PBS. $10 \%$ FBS in PBS was added after rinsing.

\subsection{General Animal Imaging}

All animal procedures were approved by the Institutional Animal Care and Use Committee, and the studies here were carried out in compliance with these approved procedures.

\subsection{In Vivo Imaging}

Briefly, $10^{5}$ MDA-MB-231 luc-D3H2LN cells were injected under the skin on the flank of a nude mouse (two tumors per mouse, eight tumors per reagent studied). After $\sim 3$ weeks of growth, animals were chosen for use when their tumor was $\sim 6 \times 6 \times 3 \mathrm{~mm}^{3}$ in size. The CELSI scan was completed vertically. Images of the luminescence at different delay times between LINAC pulse and phosphorescence emission were acquired to construct emission lifetime. Under general anesthesia of inhaled isofluorane, $50 \mu \mathrm{L}$ of $50 \mu \mathrm{M}$ of each reagent was directly injected into the tumors and the animal was imaged alive and then again at $30 \mathrm{~min}$ after euthanize, which allows determination of emission lifetime at ambient $\mathrm{pO}_{2}$ (alive) low $\mathrm{pO}_{2}$ environments (dead), respectively.

\subsection{Statistical Analysis}

The tissue $\mathrm{pO}_{2}$ was determined utilizing the Stern-Volmer relationship. The differences between the live and dead conditions, as shown in Fig. 5 with $n=8$ paired samples each, were assessed using a two-tailed Students $t$-test, with $\alpha=0.05$ and resulting $P<0.001$. This shows that there are significant differences between the values of both emission lifetime and 

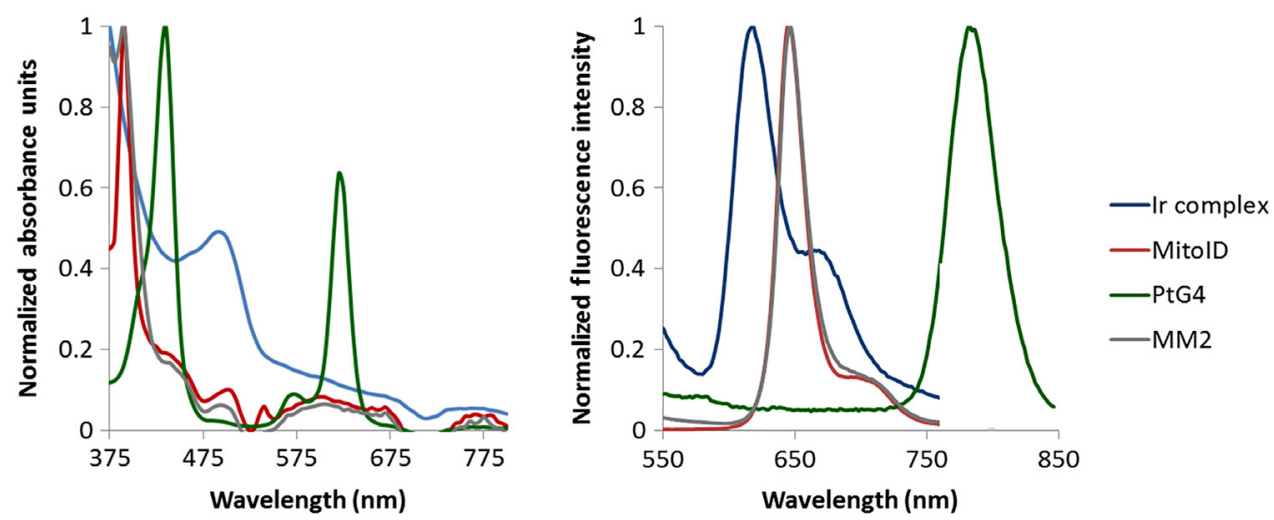

Fig. 1 Absorbance and emission spectra for oxygen sensors. The absorbance and fluorescence emission spectra for $\operatorname{Ir}(\mathrm{btb})_{2}(\mathrm{acac})$ (blue), MitolD (red), PtG4 (green), and MM2 (gray).

(a)

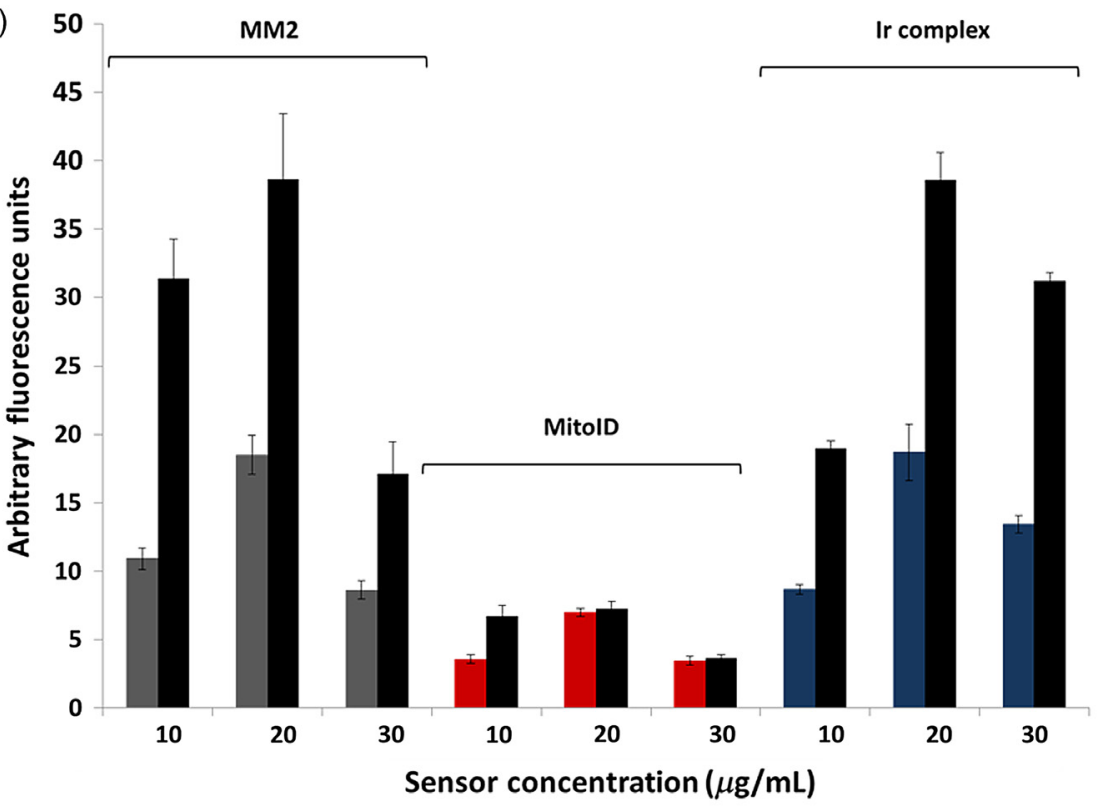

(b)
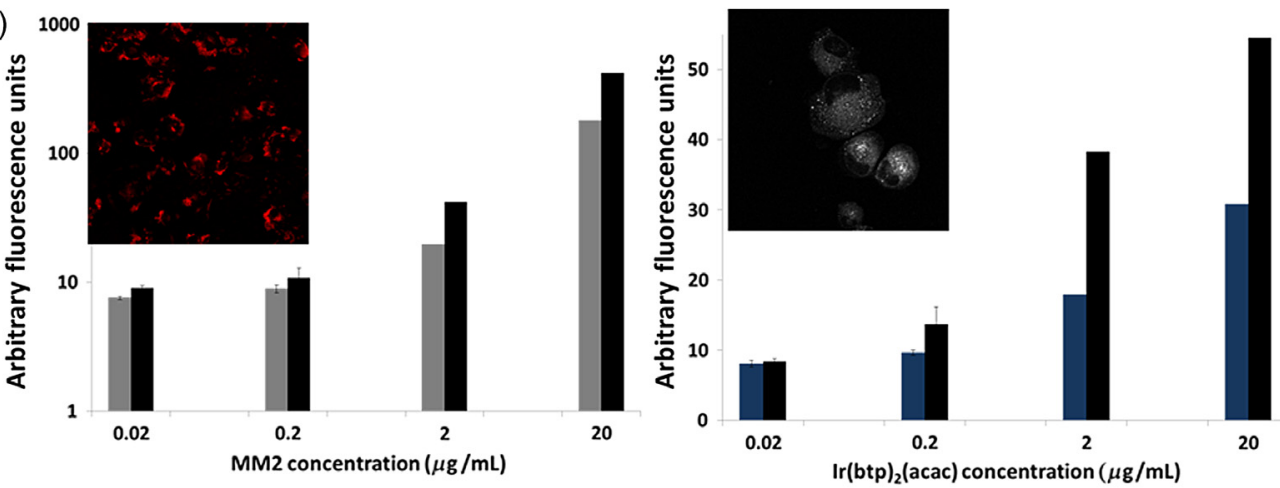

Fig. 2 Comparison of commercially available oxygen sensors in PANC-1 cells. (a) MM2 (gray), MitolD (red), and $\operatorname{Ir}(\mathrm{btb})_{2}$ (acac) (blue) were incubated with PANC-1 cells overnight. Fluorescence was measured in the presence of ambient oxygen [light gray, MM2, medium gray, MitolD, and dark gray, $\left.\operatorname{Ir}(\mathrm{btb})_{2}(\mathrm{acac})\right]$ and with glucose oxidase catalase enzymatic oxygen scavenging (black). (b) The brightest commercial oxygen sensors were explored at decreasing concentrations $(20,2,0.2$, and $0.02 \mu \mathrm{g} / \mathrm{mL})$ to determine the lowest concentration that can be detected. Inset shows fluorescence microscopy of each sensor $(20 \mu \mathrm{g} / \mathrm{mL})$ loaded into PANC-1 cells. Excitation and emission were measured at 380 and $660 \mathrm{~nm}$, respectively. The fluorescence intensity represents the average of six experiments \pm SEM. 
$\mathrm{pO}_{2}$ of the animals alive versus dead. Figure 5 was generated using Python 3.4.3 with the library matplotlib 2.0.0.

\section{Results}

The set of four phosphorescent oxygen sensors were directly compared to determine which of these reagents would be ideal for use in CELSI in tumors. These agents were chosen based upon published data, and they represented the most promising mix of agents for use in vivo with longer lifetime emission from phosphorescence. The absorbance and emission spectra of PtG4 (green), MM2 (gray), $\operatorname{Ir}(\text { btb })_{2}$ (acac) (blue), and MitoID (red) are shown in Fig. 1. ${ }^{25,26}$ Relative brightness of the commercially available MM2, $\operatorname{Ir}(\mathrm{btb})_{2}(\mathrm{acac})$, and MitoID was assessed at increasing concentrations $(10,20$, and $30 \mu \mathrm{g} / \mathrm{mL})$ in PANC-1 cells in the presence and absence of ambient oxygen, through the addition of the enzymatic oxygen-scavenging system of glucose oxidase and catalase (GODCAT). Measurements of emission were taken via fluorescence plate reader. MM2 and $\operatorname{Ir}(\mathrm{btb})_{2}(\mathrm{acac})$ exhibited the maximum signal under deoxygenated conditions at $20 \mu \mathrm{g} / \mathrm{mL}$, and the MitoID intensity in the presence and absence of oxygen scavengers was lower in comparison [Fig. 2(a)]. Statistically significant changes in oxygenation can be detected at concentrations as low as $0.2 \mu \mathrm{g} / \mathrm{mL}$ for $\operatorname{Ir}(\mathrm{btb})_{2}(\mathrm{acac})$ and $0.02 \mu \mathrm{g} / \mathrm{mL}$ for MM2 [Fig. 2(b)], as assessed by a student's t-test.

We sought to evaluate the effectiveness of these reagents to be imaged with Cherenkov excited luminescence (CEL). Solutions of PtG4, MM2, $\operatorname{Ir}(\mathrm{btb})_{2}$ (acac), and MitoID at decreasing concentrations $(20,2,0.2$, and $0.02 \mu \mathrm{g} / \mathrm{mL})$ were placed in a 96-well plate with and without GODCAT and evaluated via epiluminescence. The background corrected phosphorescence intensities from this experiment at the maximum concentration of $20 \mu \mathrm{g} / \mathrm{mL}$ are as follows (first number for each compound represents ambient oxygen, second number represents GODCAT): MM2 (565, 1349); PtG4 (230, 1269); $\operatorname{Ir}(\mathrm{btb})_{2}$ (acac) (45.7, 168); MitoID (119, 150) [Fig. 3(a)]. This experiment was repeated by loading all of these reagents into PANC-1 cells with and without GODCAT and imaging via CEL. The phosphorescence intensities from this experiment at the maximum concentration of $20 \mu \mathrm{g} / \mathrm{mL}$ are as follows (first number for each compound represents the signal under ambient oxygen, and the second number is the signal deoxygenated with GODCAT): MM2 (153, 376); PtG4 (181, 406); $\operatorname{Ir}(\mathrm{btb})_{2}$ (acac) (235, 633); MitoID (223, 288) [Fig. 3(b)].

A murine experiment to sense tissue oxygenation was completed in mice with subcutaneous MDA-MB-231lucD3H2LNtumors. The setup for CELSI is shown in Figs. 4(a) and 4(b). Briefly, gantry head of the linear accelerator that delivers the radiation is positioned below the mouse, which is located on the treatment couch. A mirror is placed that redirects the photons to the ICCD camera for imaging. A total of four tumors per compound were imaged, with local injection of $50 \mu \mathrm{L}$ of each reagent [50 $\mu \mathrm{M} \mathrm{PtG} 4,2.5 \mathrm{nmol}, 1.75 \mathrm{mg}$ per tumor and $50 \mu \mathrm{M}$ $\operatorname{Ir}(\mathrm{btb})_{2}$ (acac), $2.5 \mathrm{nmol}, 35 \mu \mathrm{g}$ per tumor]. Each mouse was imaged while alive and then repeated $30 \mathrm{~min}$ after euthanasia to capture both normoxic and hypoxic conditions, respectively. In the euthanized animal, the drop in blood circulation and respiration causes a dramatic decrease in $\mathrm{pO}_{2}$ values. Temperature was controlled using a heating pad under each animal, throughout the study, and the CELSI scan was completed vertically for these cases. Images of the luminescence were captured at different delay times after the LINAC pulse to determine emission lifetimes for each reagent. The maximum intensity projection images of CELSI from both PtG4 and $\operatorname{Ir}(\mathrm{btb})_{2}(\mathrm{acac})$ at a delay time of $4.26 \mu$ s are shown in Fig. 4(c). A comparison of the intensity differences between PtG4 phosphorescence at various delay times in an alive and dead mouse are shown in Fig. 5(a). The emission lifetimes were mapped for the alive and euthanized mouse for PtG4 and are quantified and displayed via a box and whiskers plot [Fig. 5(b)]. Using the Stern-Volmer equation, we estimated the average tissue $\mathrm{pO}_{2}$ with the reported quenching constant for $\mathrm{PtG} 4^{27}$ [Fig. 5(c)]. The phosphorescence at different delay times for a euthanized mouse is shown for $\operatorname{Ir}(\text { btb })_{2}$ (acac) [Fig. 5(d)]. In addition, we created a map of emission lifetimes and estimated the average $\mathrm{pO}_{2}$ using the reported quenching constant for $\operatorname{Ir}(\mathrm{btb})_{2}$ (acac) using the Stern-Volmer relationship. $^{14}$

\section{Discussion}

Due to the redshifted emission of PtG4 (772 nm) in comparison to the other reagents, it was originally hypothesized that this reagent would be the most effective for imaging in tumors
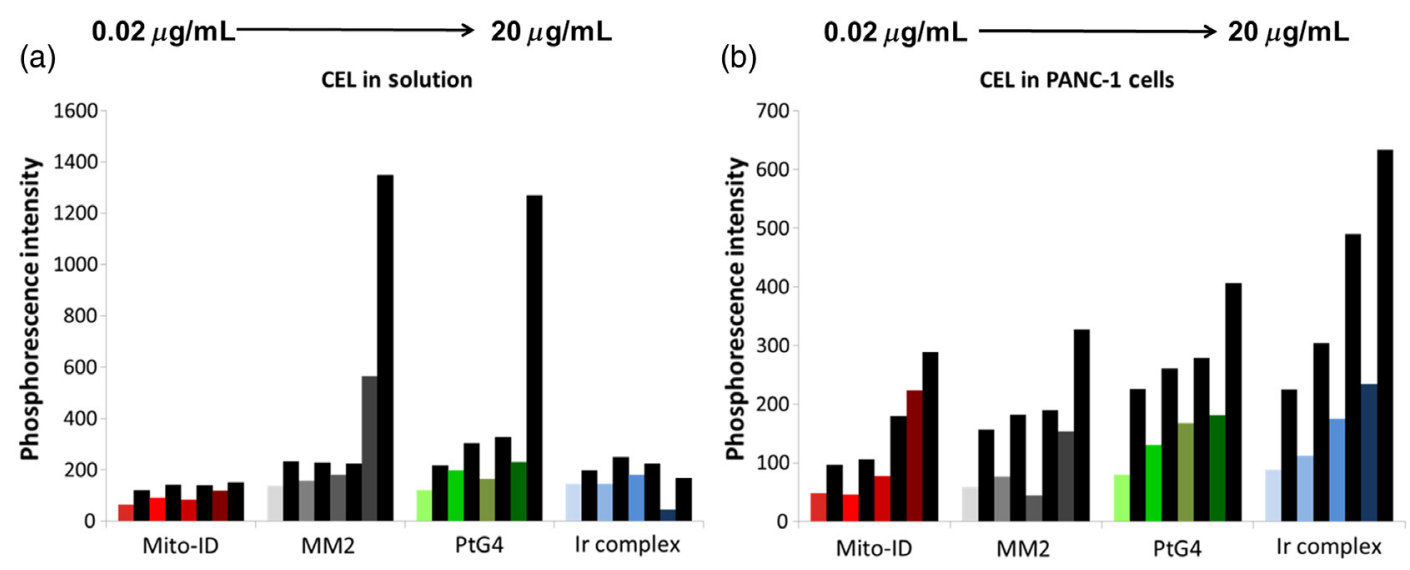

Fig. 3 Comparison of CEL with phosphorescent oxygen sensors. Increasing concentrations of each sensor indicated by darker shades of each color [0.02, 0.2, 2, and $20 \mu \mathrm{g} / \mathrm{mL}$, MitolD (red), MM2 (gray), PtG4 (green), and $\operatorname{Ir}(\mathrm{btb})_{2}(\mathrm{acac})$ (blue)] were analyzed in ambient oxygen or with enzymatic oxygen scavenger glucose oxidase/catalase (black). A 96-well plate with these reagents in (a) solution or (b) loaded into PANC-1 cells was exposed to 6-MV radiation from a LINAC, and the phosphorescence intensity was measured via ICCD. 


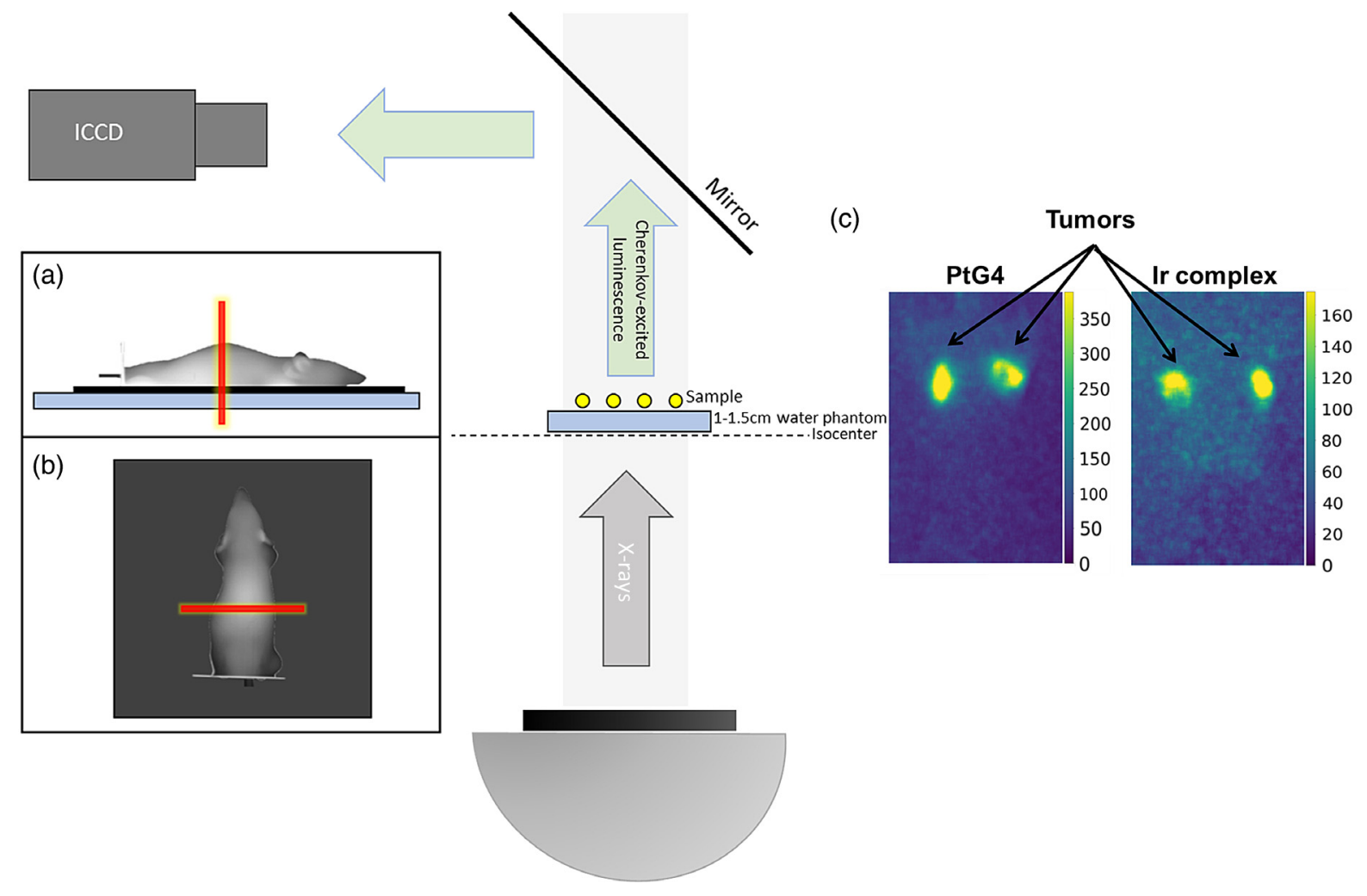

Fig. 4 CELSI setup and in vivo imaging with PtG4 and $\operatorname{Ir}(\mathrm{btb})_{2}(\mathrm{acac})$. The LINAC gantry head that delivers radiation is placed below the mouse that is positioned on the treatment couch. A mirror above redirects the photons to the ICCD. (a) Lateral view of the mouse depicting passage of radiation sheet in red (b) aerial view as imaged from the ICCD camera. (c) Under anesthesia of isofluorane, $2.5 \mathrm{nmol}$ of each reagent was injected into subcutaneous MDA-MB-231luc-D3H2LN tumors. Maximum intensity projection of PtG4 and of $\operatorname{Ir}(\mathrm{btb})_{2}(\mathrm{acac})$ at $4.26 \mu$ s delay time, respectively. Tumors are indicated with line arrows.

since this wavelength would achieve the most effective tissue penetration. However, we wanted to compare PtG4 with other commercially available phosphorescent oxygen sensors to identify the reagents with the best qualities for utilization in CELSI. Several factors are involved in the selection of ideal compounds. As with fluorescence imaging, the quantum yield of the compound is a significant factor that determines the efficiency of imaging. In CEL imaging, time gating is used to separate Cherenkov emissions from luminescence, so luminescence lifetime is also important to consider. Medical LINACs have a $4-\mu$ s radiation pulse, and so this moderate pulse time limits the time gating to the microsecond regime, and without significant change to the LINAC or acquisition, nanosecond lifetimes would not be possible to measure. The luminescent agents that are available with microsecond lifetimes are typically phosphorescent. It has not been feasible to detect fluorescent agents that have nanosecond lifetimes, other than through wavelength filtering and continuous wave detection. ${ }^{21}$ The typical timing used for image acquisition that is coupled to the LINAC is shown in Fig. 6(a). Experimentally, the gate width is generally set between 5 and 10 times the deoxygenated lifetime of the reagent of interest $\left(\tau_{0}\right)$. The CEL is then detected at multiple delay times in order to determine the emission lifetime of each phosphorescent agent. The time between radiation pulses is one factor that will limit the maximum lifetime when choosing a phosphorescent compound. The LINAC generates radiation pulses at a repetition rate between 2.7 and $17 \mathrm{~ms}$ (600 to $100 \mathrm{MU} / \mathrm{min}$ ). Shorter lifetimes can be difficult to discriminate from the radiation-induced Cherenkov light. The LINAC radiation pulse does not immediately turn off, and both stray charge and Cherenkov emissions may be detected during the transition phase. Phosphorescent compounds with lifetimes in this range $(<4.5 \mu \mathrm{s})$ will be difficult to detect. A plot of the decay curves depicting lifetime measurements as a function of quantum yield for each compound used in the study is shown in Fig. 6(b). $\operatorname{Ir}(\mathrm{btb})_{2}(\mathrm{acac})$ has been utilized in LED applications and has a high quantum yield of $0.33,{ }^{26}$ which should also impart ideal imaging properties; however, its shorter emission lifetime of $5.8 \mu \mathrm{s}^{26}$ may limit its application in CELSI. While PtG4, MM2, and MitoID have lower quantum yields, their longer emission lifetimes should be advantageous for signal detection. In addition, MM2 and MitoID are nanoparticles with multiple copies of oxygen-sensitive porphyrins, ${ }^{25}$ which should provide enhanced signal-to-noise ratio.

With optimization in mind, a comparison of commercially available oxygen sensors was completed in vitro, comparing signals in ambient oxygen to that in the presence of oxygen-scavenging GODCAT. These conditions were employed since phosphorescent oxygen sensors exhibit maximum luminescence in the absence of oxygen. PtG4 was not employed in this study since the maximum cutoff for the fluorescence emission of the plate reader is shorter than the PtG4 fluorescence emission. As expected, these reagents show a signal enhancement in the presence of GODCAT conditions, indicating a response to change in oxygenation levels. MM2 and $\operatorname{Ir}(\mathrm{btb})_{2}(\mathrm{acac})$ gave the most robust responses in sensing oxygen changes via steady state 
(a)

Alive
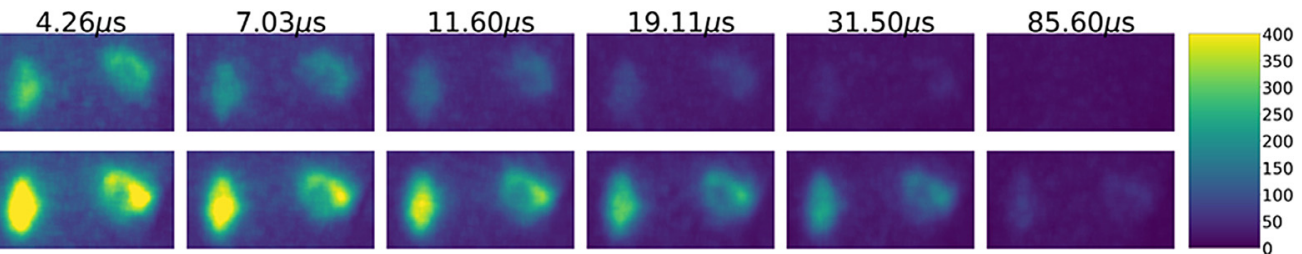

(b)
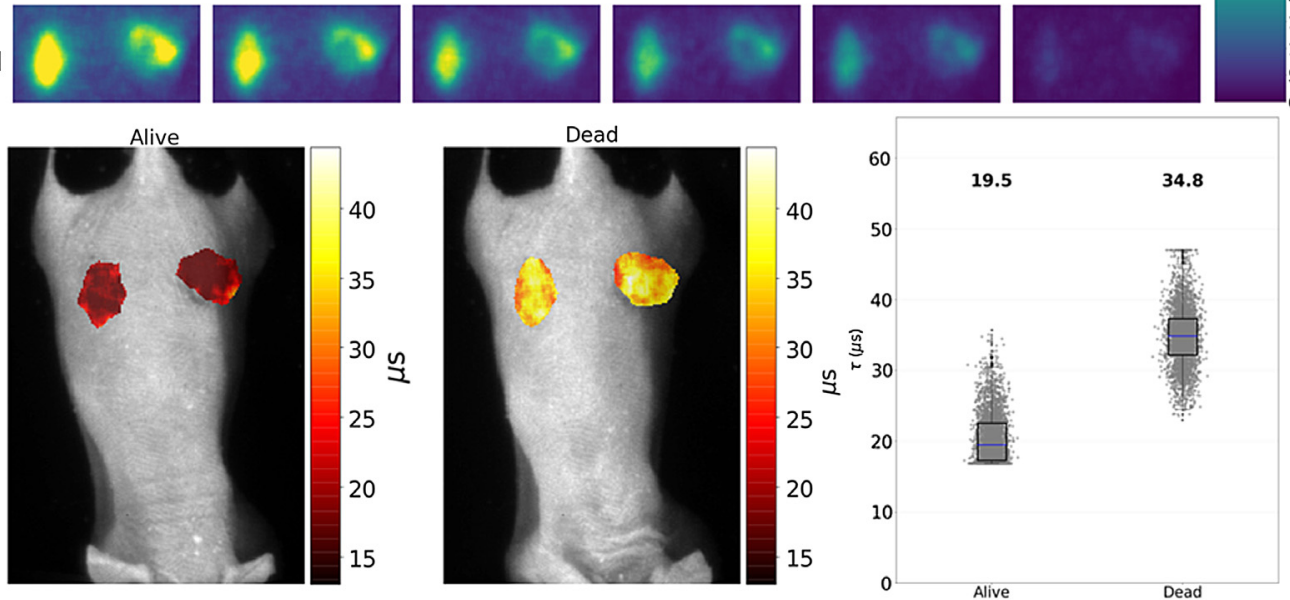

(c)
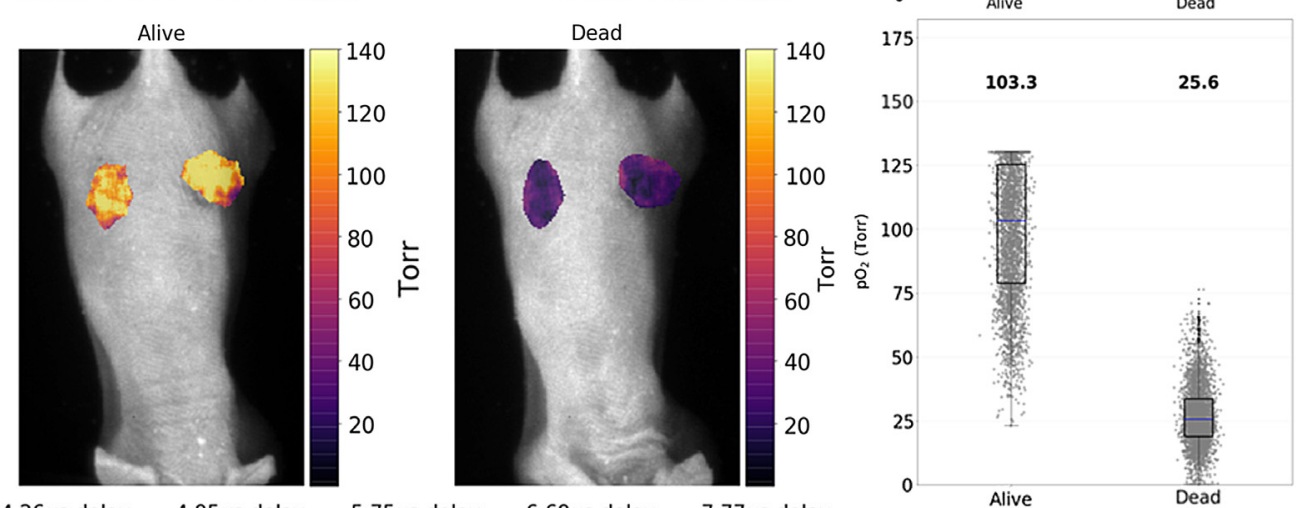

(d)
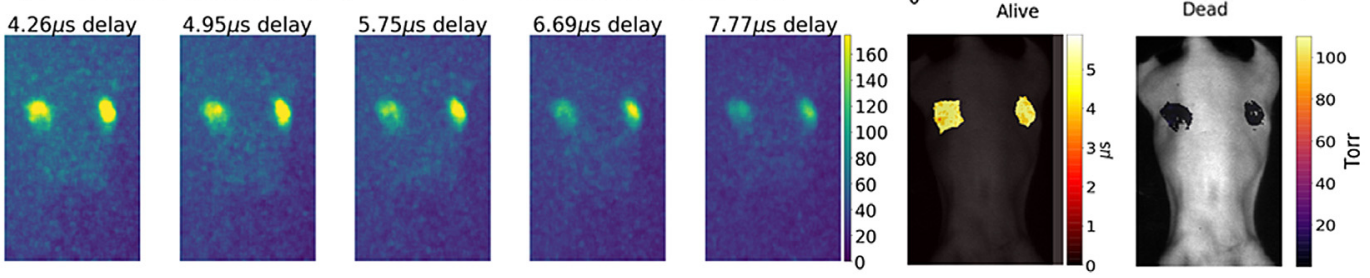

Fig. 5 In vivo lifetime imaging with PtG4 and $\operatorname{Ir}(\mathrm{btb})_{2}$ (acac). Under anesthesia of isofluorane, the animal was imaged at various delay times based on reported lifetimes of each reagent to gain emission lifetime information alive and $30 \mathrm{~min}$ after euthanize, when the drop in blood circulation and respiration causes a marked decrease in $\mathrm{pO}_{2}$ values. (a) Comparison of PtG4 phosphorescence intensity at various delay times based on known PtG4 emission lifetime in subcutaneous tumors alive (top) and 30 min after euthanize(bottom). (b) PtG4 emission lifetime maps and box and whiskers plot of emission lifetimes of alive and euthanized mouse. (c) $\mathrm{pO}_{2}$ maps and box and whiskers plot of oxygen levels in alive and euthanized mouse for PtG4. (d) Phosphorescence intensity of tumors injected with $\operatorname{Ir}(\mathrm{btb})_{2}(\mathrm{acac})$ at different delay times informed from the reported $\operatorname{Ir}(\mathrm{btb})_{2}$ (acac) emission lifetime, map of emission lifetime, and $\mathrm{pO}_{2}$ map in euthanized mouse. All lifetime maps and subsequent $\mathrm{pO}_{2}$ maps for both reagents were constructed utilizing data from the $4.26-\mu$ s delay time.

fluorescence [Fig. 2(a)]. At the highest concentration utilized $(30 \mu \mathrm{g} / \mathrm{mL})$, the signal was attenuated in comparison to the $20 \mu \mathrm{g} / \mathrm{mL}$ samples, which could be attributed to self-quenching, although further investigation of this was not done. Due to these observations, since MM2 and $\operatorname{Ir}(\mathrm{btb})_{2}(\mathrm{acac})$ gave the best signal, the next focus was to determine the lowest concentration of each sensor that could be utilized to detect oxygen levels in vitro. We found that $\operatorname{Ir}(\mathrm{btb})_{2}(\mathrm{acac})$ still elicited a luminescence signal in response to oxygen changes in PANC-1 cells at $0.2 \mu \mathrm{g} / \mathrm{mL}$. While MM2 exhibited a statistically significant response to oxygen scavenging conditions at a lower concentration $(0.02 \mu \mathrm{g} / \mathrm{mL})$, the responses to oxygenation changes were not robust at concentrations lower than $2 \mu \mathrm{g} / \mathrm{mL}$ [Fig. 2(b)].

We investigated CEL both in solution and loaded into PANC1 cells of the previously studied PtG4 as well as MM2, $\operatorname{Ir}(\mathrm{btb})_{2}$ (acac), and MitoID. Both PtG4 and MM2 exhibited strong phosphorescence at $20 \mu \mathrm{g} / \mathrm{mL}$ in solution, particularly with oxygen scavenging, when excited by Cherenkov emission, with phosphorescence intensities of 1349 and 1269, respectively, whereas $\operatorname{Ir}(\mathrm{btb})_{2}(\mathrm{acac})$ and MitoID did not display significant phosphorescence in solution, with intensities of 250 and 
(a)

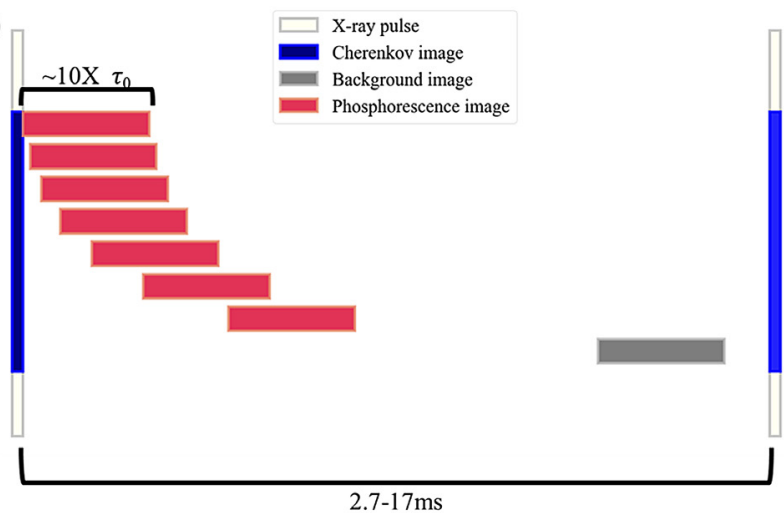

(b)

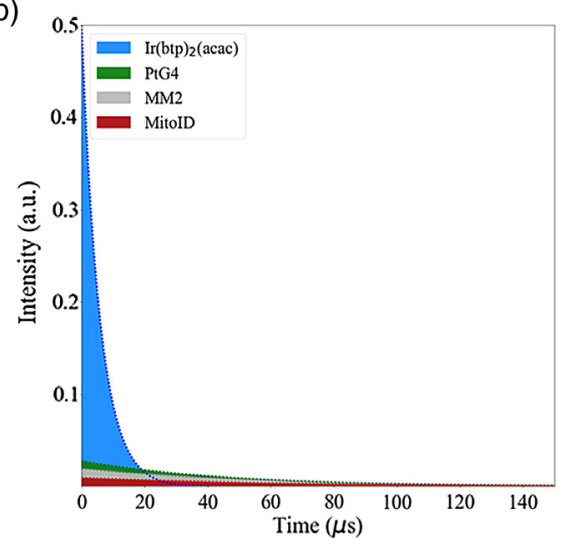

Fig. 6 Considerations for CELSI. (a) Cherenkov imaging timing. Experimentally, the gate width is generally set between 5 and 10 times the deoxygenated lifetime $\left(\tau_{0}\right)$ of the reagent of interest. Luminescence is detected at a series of delay times (red blocks) after the Cherenkov emission (blue column) to assess the emission lifetime of phosphorescent agents. The LINAC generates radiation pulses at a repetition rate between 2.7 and $17 \mathrm{~ms}(600$ to $100 \mathrm{MU} / \mathrm{min}$ ). (b) Plot of emission decay curves as a function of quantum yield.

150, respectively [Fig. 3(a)]. We have previously seen excellent results with CELSI with PtG4,,$^{19,21,22}$ and since MM2 contains multiple copies of a porphyrin grafted to a nanoparticle, enhanced signal in response to oxygen was anticipated for both of these reagents. As expected, we found that the intracellular phosphorescence intensity was markedly decreased in comparison to the phosphorescence measured in solution. Interestingly, $\operatorname{Ir}(\mathrm{btb})_{2}$ (acac) exhibits a much better phosphorescence signal in cells versus solution, giving the largest detectable signal in comparison to the other reagents [Fig. 3(b)]. This is likely due to the fact that this complex binds to albumin or another cellular protein, thereby enhancing permeability. However, previous reports indicate that it is no longer sensitive to oxygen when bound to albumin in vitro. Our results and other cell studies with $\operatorname{Ir}(\mathrm{btb})_{2}(\mathrm{acac})$ that show oxygen sensitivity indicate that binding to albumin is not the mechanism that imparts enhanced signal in cells versus solution. ${ }^{26}$ The performance of MM2 was diminished intracellularly, possibly due to lower cell permeability than $\operatorname{Ir}(\mathrm{btb})_{2}(\mathrm{acac})$. In fact, $\operatorname{Ir}(\mathrm{btb})_{2}(\mathrm{acac})$ was found to penetrate cells far more rapidly than MM2 (30 min versus $24 \mathrm{~h}$ ). PtG4 gave a robust response to oxygenation changes in cells; however, the intensity was somewhat attenuated in comparison to $\operatorname{Ir}(\mathrm{btb})_{2}(\mathrm{acac})$. One critical advantage of PtG4 is that the dendrimer shell prevents its
Table 1 Side-by-side comparison of phosphorescent oxygen sensors utilized for CEL.

\begin{tabular}{lcccccc} 
& \multicolumn{2}{c}{ CEL } & CEL CELSI Biomolecule & & Self- \\
Compound & solution & cells & in vivo & interaction & Stability quenching \\
\hline PtG4 & + & + & + & - & + & - \\
$\operatorname{Ir}(\text { btb })_{2}($ acac $)$ & + & + & + & + & + & + \\
MM2 & + & + & - & - & - & + \\
MitolD & - & + & - & + & - & + \\
\hline
\end{tabular}

interaction with other biomolecules that could potentially perturb its emission lifetime and thus $\mathrm{pO}_{2}$ estimations. This is considered to be a key factor in making this agent a linear reporter of tissue $\mathrm{pO}_{2}$, and in fact PtG4 and similar reagents have been calibrated appropriately in extensive studies. ${ }^{19-24,27}$ While MitoID displays an enhanced signal in cells versus solution, it still elicits the weakest phosphorescent response to changes in oxygen concentration.

PtG4, $\operatorname{Ir}(\mathrm{btb})_{2}$ (acac), and MM2 seemed to have promise for use in CELSI. Therefore, we investigated these reagents for sensing oxygenation levels in subcutaneous tumors. We were unable to detect significant phosphorescence with MM2 in vivo. However, we were able to sense emission lifetime and as such calculate tissue oxygenation utilizing CELSI for both PtG4 and $\operatorname{Ir}(\mathrm{btb})_{2}(\mathrm{acac})$ at the nmol level [Figs. 5(b)-5(d)]. Unfortunately, we were not able to measure fluorescence lifetime or tissue $\mathrm{pO}_{2}$ in live mice with $\operatorname{Ir}(\mathrm{btb})_{2}(\mathrm{acac})$. This is likely because the luminescence lifetime in a fully oxygenated sample of $\operatorname{Ir}(\mathrm{btb})_{2}(\mathrm{acac})$ is shorter than we can detect since the shortest delay time feasible for imaging after the radiation pulse is $4.26 \mu \mathrm{s}$. We also observed quenching with $\operatorname{Ir}(\mathrm{btb})_{2}(\mathrm{acac})$ at concentrations higher than $50 \mu \mathrm{M}$ in subcutaneous tumors, as we did in the fluorescence plate reader experiments.

A summary of the results as well as a comparison of the pros and cons of the oxygen sensors we utilized for this study are provided in Table 1. Briefly, both PtG4 and $\operatorname{Ir}(\mathrm{btb})_{2}(\mathrm{acac})$ are stable indefinitely, whereas MM2 and MitoID nanoparticles have a short shelf life of only 1 to 2 weeks once resuspended. $\mathrm{PtG} 4$ has a dendrimer shell that prevents its interaction with biomolecules, allowing accurate determinations of fluorescence lifetime and $\mathrm{pO}_{2}$ calculations. $\operatorname{Ir}(\mathrm{btb})_{2}(\mathrm{acac})$ and MitoID appear to interact with biomolecules in some way, given the difference in phosphorescent intensities discovered in solution versus cells. Finally, we have observed that MM2, MitoID, and $\operatorname{Ir}(\mathrm{btb})_{2}(\mathrm{acac})$ exhibit self-quenching at higher concentrations, where we have not seen this effect with increasing concentrations of PtG4.

\section{Conclusion}

When used in vitro in PANC-1 cells at the same concentrations, the signal strengths suggest that the optimal agents would be $\operatorname{Ir}(\mathrm{btb})_{2}(\mathrm{acac})$, followed by PtG4, and then MM2 and MitoID. However, in vivo, only $\mathrm{PtG} 4$ and $\operatorname{Ir}(\mathrm{btb})_{2}(\mathrm{acac})$ were found to be measurable at nmol doses in the tumors, and assessment of tissue oxygenation accomplished. On the timescale of LINAC produced $\mathrm{x}$-ray imaging, PtG4 is the more ideal agent because of the lifetime on the scale of tens of microseconds versus $\operatorname{Ir}(\mathrm{btb})_{2}(\mathrm{acac})$, which has a lifetime 
of a few microseconds, which hinders detection at higher tissue $\mathrm{pO}_{2}$ values. This work represents: (1) a unique way to harness Cherenkov emission for imaging purposes in conjunction with radiotherapy, (2) a noninvasive determination of tumor $\mathrm{pO}_{2}$, and (3) a direct comparison of phosphorescent sensors available to probe tissue oxygenation.

\section{Disclosures}

The authors report no conflicts of interest.

\section{Acknowledgments}

This work was financially supported by the Department of Defense, Congressionally Directed Medical Research Program (CDMRP) Breast Cancer Innovator Award (BC150584P1), as well as the National Institutes of Health NIBIB R01 EB024498, and the NCI Cancer Center Support Grant 5P30CA023108-37. This material was also based on work supported by the National Science Foundation Graduate Research Fellowship.

\section{References}

1. P. Vaupel, A. Mayer, and M. Hockel, "Impact of hemoglobin levels on tumor oxygenation: the higher, the better?" Strahlenther. Onkol. 182(2), 63-71 (2006).

2. S. M. Evans and C. J. Koch, "Prognostic significance of tumor oxygenation in humans," Cancer Lett. 195(1), 1-16 (2003).

3. J. Bussink et al., "Effects of nicotinamide and carbogen on oxygenation in human tumor xenografts measured with luminescense based fiberoptic probes," Radiother. Oncol. 57(1), 21-30 (2000).

4. A. Turaka et al., "Hypoxic prostate/muscle ratio predicts for outcome in patients with localized prostate cancer: long-term results," Int. J. Radiat. Oncol. Biol. Phys. 82(3), e433-e439 (2012).

5. P. Vaupel and D. K. Kelleher, "Blood flow and oxygenation status of prostate cancers," Adv. Exp. Med. Biol. 765, 299-305 (2013).

6. O. Genbacev et al., "Human cytotrophoblast expression of the von Hippel-Lindau protein is downregulated during uterine invasion in situ and upregulated by hypoxia in vitro," Dev. Biol. 233(2), 526536 (2001).

7. C. J. Koch, "A two-component assay for hypoxia incorporating longterm nitroreduction and short-term DNA-damage allows differentiation of the three hypoxia sub-types," Radiat. Res. 190(1), 72-87 (2018).

8. S. K. Loftus et al., "Hypoxia-induced HIF1 $\alpha$ targets in melanocytes reveal a molecular profile associated with poor melanoma prognosis," Pigm. Cell Melanoma Res. 30(3), 339-352 (2017).

9. H. B. Stone and H. R. Withers, "Tumor and normal tissue response to metronidazole and irradiation in mice," Radiology 113(2), 441-444 (1974).

10. K. Y. Aguilera and R. A. Brekken, "Hypoxia studies with pimonidazole in vivo," Bio-Protocol 4(19), e1254 (2014).
11. M. R. Gonçalves et al., "Decomposition of spontaneous fluctuations in tumour oxygenation using BOLD MRI and independent component analysis," Br. J. Cancer 113(8), 1168-1177 (2015).

12. D. Li et al., "Correlation between BOLD-MRI and HIF expression level in renal carcinoma," Int. J. Clin. Exp. Pathol. 8(10), 13759-13763 (2015).

13. H. Gustafsson et al., "EPR oximetry of cetuximab-treated head-andneck tumours in a mouse model," Cell Biochem. Biophys. 75(3-4), 299-309 (2017).

14. H. M. Swartz, The measurement of oxygen in vivo using EPR techniques, in In Vivo EPR (ESR): Theory and Application, L. J. Berliner, Ed., pp. 403-440, Springer US, Boston, Massachusetts (2003).

15. S. Srinivasan et al., "In vivo hemoglobin and water concentrations, oxygen saturation, and scattering estimates from near-infrared breast tomography using spectral reconstruction," Acad. Radiol. 13(2), 195-202 (2006).

16. O. J. Kelada et al., "Quantification of tumor hypoxic fractions using positron emission tomography with [(18)F]fluoromisonidazole ([(18) F]FMISO) kinetic analysis and invasive oxygen measurements," Mol. Imaging Biol. 19(6), 893-902 (2017).

17. S. Stieb et al., "Longitudinal PET imaging of tumor hypoxia during the course of radiotherapy," Eur. J. Nucl. Med. Mol. Imaging 45(12), 22012217 (2018)

18. A. K. Glaser et al., "Projection imaging of photon beams by the Cerenkov effect," Med. Phys. 40(1), 12101 (2013).

19. R. Zhang et al., "Cerenkov radiation emission and excited luminescence (CREL) sensitivity during external beam radiation therapy: Monte Carlo and tissue oxygenation phantom studies," Biomed. Opt. Express 3(10), 2381-2394 (2012).

20. R. Zhang et al., "Superficial dosimetry imaging based on Cerenkov emission for external beam radiotherapy with megavoltage x-ray beam," Med. Phys. 40(10), 101914 (2013).

21. H. Lin et al., "Comparison of Cherenkov excited fluorescence and phosphorescence molecular sensing from tissue with external beam irradiation," Phys. Med. Biol. 61(10), 3955-3968 (2016).

22. R. Zhang et al., "Oxygen tomography by Cerenkov-excited phosphorescence during external beam irradiation," J. Biomed. Opt. 18(5), 50503 (2013).

23. T. V. Esipova et al., "Two new 'protected' oxyphors for biological oximetry: properties and application in tumor imaging," Anal. Chem. 83(22), 8756-8765 (2011).

24. R. I. Dmitriev and D. B. Papkovsky, "Intracellular probes for imaging oxygen concentration: how good are they?" Methods Appl. Fluoresc. 3(3), 34001 (2015).

25. R. I. Dmitriev and D. B. Papkovsky, "Optical probes and techniques for $\mathrm{O}_{2}$ measurement in live cells and tissue," Cell Mol. Life Sci. 69(12), 2025-2039 (2012).

26. S. Zhang et al., "Phosphorescent light-emitting iridium complexes serve as a hypoxia-sensing probe for tumor imaging in living animals," Cancer Res. 70(11), 4490-4498 (2010).

27. C. T. Sullender et al., "Imaging of cortical oxygen tension and blood flow following targeted photothrombotic stroke," Neurophotonics 5(3), 035003 (2018).

Biographies of the authors are not available. 